Publ. RIMS, Kyoto Univ.

12 Suppl. (1977), 5-17.

\title{
Propagation of Analytic and Differentiable Singularities for Solutions of Partial Differential Equations
}

\author{
by \\ Jean-Michel BoNY*
}

In the first part of this paper, we study propagation of singularities for solutions of an analytic pseudo-differential equation, the characteristic set of which is a regular involutive manifold. There exists a natural foliation of this manifold, and (theorem 1.7) the analytic singular spectrum of a solution is a union of leaves-this is a joint work with P. Schapira. The same result holds for differentiable singular spectrum (wave front) assuming Levi's condition. A similar result had been proved by J. Sjöstrand for $C^{\infty}$ pseudo-differential equations, but with additional assumptions on complex characteristics [14]. We prove also microlocal solvability for our operators (theorem 1.6). Complete proofs are given in [6] and [4].

In the second part of this paper ( $\S 4$ to 7 ), we study operators the characteristic set of which contains a regular involutive manifold. The main result (theorem 5.6) is an analogue, for singular spectrum, to Holmgren theorem for support. Applications to propagation of analytic singularities and to uniqueness of Cauchy problem are given. Besides arguments used in the first part, we use results of Kashiwara [11], [12] and direct infinitesimal geometry. A more detailed exposition is given in [5].

1. Propagation of Analytic and Differentiable Singularities When the Characteristic Set is an Involutive Manifold

Received September 22, 1976.

* Université de Paris-Sud, 91405 Orsay, France. 


\section{$\S 1$. Main Theorems}

Let $P\left(x, D_{x}\right)$ be an analytic pseudo-differential operator of order $\mu$, defined near a point $\left(x_{0}, \xi_{0}\right) \in \mathbb{R}^{\nu} \times S^{\nu-1}$. Let $P_{\mu}$ be its principal symbol and $\operatorname{Char}(P)=\left\{(x, \xi) ; P_{\mu}(x, \xi)=0\right\}$ its characteristic set. We shall use following assumptions on $P$

1.1. Char $(P)$ is a regular involutive manifold, of codimension $n$.

That means that $\operatorname{Char}(P)$ can be defined by analytic equations

$$
q_{1}(x, \xi)=\cdots=q_{n}(x, \xi)=0
$$

with $\left\{q_{i}, q_{j}\right\}=0$ on $\operatorname{Char}(P)$ and $d q_{1}, \cdots, d q_{n}, \Sigma \xi_{i} d x_{i}$ linearly independent on $\operatorname{Char}(P)$.

1.2. $P_{\mu}$ vanishes on $\operatorname{Char}(P)$ exactly at order $m$, i.e. for each point $(x, \xi)$ of $\operatorname{Char}(P)$ and for each vector $(\delta x, \delta \xi)$ transversal to Char $(P)$ at this point, we have:

$$
P_{\mu}(x+\varepsilon \delta x, \xi+\varepsilon \delta \xi)=a \varepsilon^{m}+o\left(\varepsilon^{m}\right) \text { with } a \neq 0 .
$$

It is then possible to find a decomposition of $P_{\mu}$ of the following type

$$
P_{\mu}(x, \xi)=\sum_{|\alpha|=m} a_{\alpha}(x, \xi) q^{\alpha}(x, \xi)
$$

with $\alpha=\left(\alpha_{1}, \cdots, \alpha_{n}\right)$, functions $a_{\alpha}$ homogeneous of degree $\mu-m$ satisfying

$$
\sum_{|\alpha|=m} a_{\alpha}(x, \xi) u^{\alpha} \neq 0 \text { for }(x, \xi) \in \operatorname{Char}(P) \text { and } u \neq 0 .
$$

The following assumption shall be used for differentiable singularities.

1.3. (Levi's condition) Let $Q_{1}, \cdots, Q_{n}$ be first order pseudo-differential operators, the principal symbols of which are functions $q_{i}$ above. Then there exist pseudo-differential operators $A_{\alpha}\left(x, D_{x}\right), 0 \leqq|\alpha| \leqq m$, of order $\mu-m$ such that

$$
P\left(x, D_{x}\right)=\sum_{0 \leqq|\alpha| \leqq m} A_{\alpha}\left(x, D_{x}\right) Q\left(x, D_{x}\right)^{\alpha}
$$

This assumption does not depend on choice of $Q_{i}$, and is merely an assumption on terms of order $\mu, \mu-1, \cdots, \mu-m+1$ in the symbol of $P$.

We shall use the two following notions to study singularities.

1.4. Analytic singular spectrum of a hyperfunction [13] We shall denote by S.S.A. $(u)$ the support of the microfunction associated to hyper- 
function $u$. If $u$ is a distribution, S.S.A. $(u)$ coincides with the essential support of $u$ defined by Bros and Iagolnitzer [1], and we shall use the following property: let $u$ be a distribution such that $\left(x_{0}, \xi_{0}\right) \notin \mathrm{S}$.S.A. (u), then $u$ is, near $x_{0}$, a sum of boundary values of holomorphic functions $f_{\alpha}$, defined near $x_{0}$ in $\mathbb{R}^{\nu}+i \Gamma_{\alpha}$ and slowly increasing (i.e. $|f(x+i y)| \leqq C|y|^{-N}$ for $y$ small) where $\Gamma_{\alpha}$ are open convex cones in $\mathbb{R}^{\nu}$ such that $\xi_{0} \notin \Gamma_{\alpha}^{0}$.

Actually, we can prove a more general result. Every notion of analytic singularity having "good properties" with respect to tensorial product, traces and integration along fibers coincides with S.S.A.. For instance, the analytic wave front defined by Hörmander for distributions [9] coincides with S.S.A..

1.5. Differentiable singular spectrum. We shall denote by S.S.D. (u) the wave front of $u$ defined by Hörmander.

1.6. Bicharacteristic leaves. Under assumption 1.1, hamiltonian fields $H_{q_{j}}$ satisfy $\left\{H_{q_{i}}, H_{q_{j}}\right\}=0$ on $\operatorname{Char}(P)$ and then define a foliation of Char $(P)$ by $n$-dimensional leaves called bicharacteristic leaves.

\subsection{Theorem (microlocal solvability)}

a) Under assumptions 1.1. and 1.2., let $v$ be a hyperfunction defined near $x_{0}$. Then, there exists a hyperfunction $u$ defined near $x_{0}$ such that $\left(x_{0}, \xi_{0}\right) \notin$ S.S.A. $(P u-v)$.

b) Under assumptions 1.1, 1.2, 1.3, let $v$ be a distribution (resp. a $C^{\infty}$ function) defined near $x_{0}$. Then, there exists a distribution (resp. a $C^{\infty}$ function) $u$ defined near $x_{0}$ such that $\left(x_{0}, \xi_{0}\right) \notin$ S.S.A. $(P u-v)$.

Remark. Strictly speaking, this statement has no meaning if $P$ is not a differential operator. But, if $P$ is a pseudo-differential operator defined near $\left(x_{0}, \xi_{0}\right), P u$ is a hyperfunction defined modulo hyperfunctions the S.S.A. of which does not contain $\left(x_{0}, \xi_{0}\right)$, so that S.S.A. $(P u-v)$ is well defined near $\left(x_{0}, \xi_{0}\right)$. The same remark holds for the following theorem.

\subsection{Theorem (Propagation of singularities)}

a) Under assumptions 1.1 and 1.2, let $u$ be a hyperfunction defined 
near $x_{0}$ satisfying $\left(x_{0}, \xi_{0}\right) \notin$ S.S.A. $(P u)$. Then, near $\left(x_{0}, \xi_{0}\right)$, the analytic singular spectrum of $u$ is a union of bicharacteristic leaves.

b) Under assumptions 1.1, 1.2, 1.3, let $u$ be a distribution defined near $x_{0}$ satisfying $\left(x_{0}, \xi_{0}\right) \notin$ S.S.D. $(P u)$. Then, near $\left(x_{0}, \xi_{0}\right)$, the differentiable singular spectrum of $u$ is a union of bicharacteristic leaves.

We shall give a sketch of the proof of theorems 1.6 and 1.7 in $\S 3$. Using a quantized contact transform, multiplying $P$ by an elliptic operator of order $m-\mu$, and changing notations, it is sufficient to prove these theorems in the following case:

$$
\nu=n+p ; \mathbb{R}^{\nu}=\mathbb{R}_{x}{ }^{n} \times \boldsymbol{R}_{t}^{p}
$$

1.8. $P\left(x, t, D_{x}, D_{t}\right)=\sum_{|\alpha|=m} A_{\alpha}\left(x, t, D_{x}, D_{t}\right) D_{x}^{\alpha}+$ lower order terms or

1.9. $P\left(x, t, D_{x}, D_{t}\right)=\sum_{0 \leq|\alpha| \leq m} A_{\alpha}\left(x, t, D_{x}, D_{t}\right) D_{x}{ }^{\alpha}$

where $A_{\alpha}$ are pseudo-differential operators of order 0 , defined for $(x, t$, $\xi, \tau)$ near $\left(0,0,0, \tau_{0}\right)$, with $\tau_{0}=(1,0, \cdots, 0)$.

\section{$\S$ 2. Pseudo-Differential Cauchy-Kowalewski Theorem in the Complex Domain}

Let us consider first a pseudo-differential operator $Q\left(z, D_{z}\right)$ of order 0 , defined for $z$ near 0 and $\xi$ near $(1,0, \cdots, 0)$. Then, we have an expansion [13]: $\quad P\left(z, D_{z}\right)=\sum a_{\alpha}(z) D_{z_{1}}^{\alpha_{1}} D_{z^{\prime}}^{\alpha^{\prime}}$, the summation being extended to multi-index $\alpha=\left(\alpha_{1}, \alpha^{\prime}\right)$ with $\alpha^{\prime} \geqq 0$ and $\alpha_{1}+\left|\alpha^{\prime}\right| \leqq 0$.

Let $\Sigma$ be the hyperplane defined by $z_{1}=\sigma$. It is then possible to define, for a holomorphic function $f$ :

$$
Q_{\Sigma} f(z)=\sum a_{\alpha}(z)\left(D_{z_{1}}^{\alpha_{1}}\right)_{\Sigma} D_{z^{\prime}}^{\alpha^{\prime}} f(z),
$$

$\left(D_{z_{1}}^{-\beta}\right)_{\Sigma} f$ being by definition the $\beta^{\text {th }}$ primitive of $f$ which vanishes at order $\beta$ on $\Sigma$ (this is closely related to action of $Q$ on holomorphic microfunctions defined in [13]).

2.1. Definition. A convex open set $\Omega$ is said $k$ - $\Sigma$-flat $(k>0)$ if 


$$
z \in \Omega, \widetilde{z} \in \Sigma,\left|z_{1}-\widetilde{z}_{1}\right| \geqq k\left|z_{i}-\widetilde{z}_{i}\right|, \quad i=2, \cdots, \nu
$$

imply

$$
\widetilde{z} \in \Omega \cap \Sigma
$$

2.2. Theorem. There exists $k>0$ such that if $f$ is holomorphic in a $k$ - $\Sigma$-flat domain $\Omega$, contained in a sufficiently small neighbourhood of $O$, then $Q_{\Sigma} f$ is holomorphic in $\Omega$.

Moreover, estimates involving growth conditions on $f$ and $Q_{\Sigma} f$ near the boundary of $\Omega$ can be given.

Let us now consider the following situation, which is the complexification of 1.8 and 1.9, after putting $P$ in Weierstrass form.

$P\left(z, w, D_{z}, D_{w}\right)$ is a pseudo-differential operator in $\mathbb{C}_{z}^{n} \times \mathbb{C}_{w}{ }^{p}$, defined for $(z, w, \zeta, \theta)$ near $\left(0,0,0, \theta_{0}\right)$, with $\theta_{0}=(1,0, \cdots, 0)$.

2.3. $P\left(z, w, D_{z}, D_{w}\right)=D_{z_{n}}^{m}+\sum_{|\alpha|=m, \alpha_{n}<m} A_{\alpha}\left(z, w, D_{z^{\prime}}, D_{w}\right) D_{z^{\prime}}^{\alpha^{\prime}} D_{z_{n}}^{\alpha_{n}}+\cdots$ or

2.4. $P\left(z, w, D_{z}, D_{w}\right)=D_{z_{n}}^{m}+\sum_{0 \leqq|\alpha| \leqq m, \alpha_{n}<m} A_{\alpha}\left(z, w, D_{z^{\prime}}, D_{w}\right) D_{z^{\prime}}^{\alpha^{\prime}} D_{z_{n}}^{\alpha_{n}}$. where $A_{\alpha}$ are of order 0 and do not depend on $D_{z_{n}}$.

2.5. Theorem. There exist $k>0$ and $\delta>0$ such that, if $\Omega$ is a convex open set, contained in a sufficiently small neighbourhood of 0 , such that each slice $\Omega \cap[w=$ constant $]$ is $\delta$-H-flat and each slice $\Omega$ $\cap\left[z_{n}=\right.$ constant $]$ is $k$ - $\Sigma$-flat, where $H$ and $\Sigma$ are hyperplanes $z_{n}=h$ and $w_{1}=\sigma$, we have:

a) Under assumption 2.3, if $g$ and $\left(h_{j}\right), j=0, \cdots, m-1$ are holomorphic respectively in $\Omega$ and in $\Omega \cap H$, there exists one and only one solution $f$ holomorphic in $\Omega$ of the Cauchy problem:

$$
\begin{aligned}
& P_{\Sigma} f=g \\
& \left.D_{z_{n}}^{j} f\right|_{H}=h_{j} \quad j=0, \cdots, m-1 .
\end{aligned}
$$

b) Under assumption 2.4 , suppose that $\Omega$ is contained in [ $\operatorname{Im} w_{1}>0$ ] 
and that $g$ and $h_{j}$ are bounded by a constant times $\left|\operatorname{Im} w_{1}\right|^{-N}$ (or that $g, h_{j}$ and all their derivatives are bounded). Then, estimates almost of the same type hold for $f$.

This theorem is proved using a successive approximation method and of course, very precise estimates are needed for operators $A_{\alpha \Sigma}$ and for commutators. These estimates in part b) are the crucial step where Levi's condition is used.

\section{§3. Sketch of the Proof of Theorems 1.6 and 1.8}

Let $G$ be an open convex cone in $\mathbb{R}^{n+p}$, the polar set $G^{\circ}$ of which is a small neighbourhood of $\xi=0, \tau=\tau_{0}=(1,0, \cdots, 0)$, and let $\Gamma$ be an open convex cone in $\mathbb{P}^{n}$ with $\Gamma^{\circ}$ sufficiently small. It is then possible to find "flat" (as in theorem 2.5) open convex subsets of $\mathbb{C}^{n+p}$ which coincide with $\mathbb{R}^{n+p}+i(G+\Gamma)$ near the origin. Using theorem 2.5, we obtain:

\subsection{Theorem。}

a) Let $g$ be holomorphic in $\mathbb{R}^{n+p}+i(G+\Gamma)$ near 0 . Then, there exists $f$ holomorphic in $\mathbb{R}^{n+p}+i\left(G^{\prime}+\Gamma\right)$, near 0 , for each $G^{\prime} \subset \subset G$, such that

$$
P b(f)=b(g) .
$$

b) Let $f$ be holomorphic in $\mathbb{R}^{n+p}+i(G+\Gamma)$ near 0 , such that $P b(f)$ $=0$. Then, $f$ can be extended as a holomorphic function in $\mathbb{R}^{n+p}+i$ $\left(G^{\prime}+\mathbb{R}^{n}\right)$ near 0 , for each $G^{\prime} \subset \subset G$.

c) Assuming Levi's condition, a) and b) hold for slowly increasing functions $f$ and $g$, and a) holds for functions all derivatives of which are bounded.

In this theorem, $b(\cdot)$ denotes the boundary value, and equality means equality as microfunctions near $\left(0, \tau_{0}\right)$.

It is now easy to prove microlocal solvability. We write $v=b(g)$ with $g$ holomorphic in $\mathbb{R}^{n+p}+i G$ near 0 . For a convenient family $\Gamma_{\alpha}$, we can write $g=\Sigma g_{\alpha}$, with $g_{\alpha}$ holomorphic in $\mathbb{R}^{n+p}+i\left(G+\Gamma_{\alpha}\right)$ near 0 , 
and then we solve $b\left(P f_{\alpha}\right)=b\left(g_{\alpha}\right)$. Putting $u=\Sigma b\left(f_{\alpha}\right)$, we obtain a microlocal solution of $P u=v$.

To prove propagation of singularities, suppose that S.S. (A. or D.) $u$ $\nexists\left(0, \tau_{0}\right)$. Using solvability, we can suppose $u=b(f)$ with $P b(f)=0$. Using a decomposition $f=\Sigma f_{\alpha}$ as above, we obtain $P b\left(f_{\alpha}\right)=b\left(g_{\alpha}\right)$ with $\Sigma b\left(g_{a}\right)=0$. Arguments similar to (and simpler than) "edge of the wedge theorem" imply that there exist $g_{\alpha \beta}$ holomorphic in $\mathbb{R}^{n+p}+i\left(G+\Gamma_{\alpha}+\Gamma_{\beta}\right)$ near 0 , with $g_{\beta \alpha}=-g_{\alpha \beta}$ and $g_{\alpha}=\Sigma g_{\alpha \beta}$. Solving $P b\left(f_{\alpha \beta}\right)=g_{\alpha \beta}$, we are reduced to $f=\Sigma f_{\alpha}{ }^{\prime}, P b\left(f_{\alpha}{ }^{\prime}\right)=0$.

Using now part b) of theorem 3.1, we obtain that $f_{\alpha}^{\prime}$ and hence $f$ are holomorphic in $\mathbb{R}^{n+p}+i\left(G^{\prime}+\mathbb{R}^{n}\right)$. We can take the partial boundary value: $u(z, t)=b_{w} f(z, w)$, which is defined on $\mathbb{C}^{n} \times \mathbb{R}^{p}$ near 0 , and satisfies $\partial / \partial \bar{z}_{j} u(z, t)=0$. Using propagation of analytic or differentiable singularities [13], [7], for partial Cauchy-Riemann system, we can then prove the propagation of singularities for $u(x, t)=b_{z} u(z, t)$.

In some sense, the argument above is the proof "with parameters $t$ and $D_{t}$ " of analyticity of solutions of elliptic equations (with respect to $x)$.

\section{H耳. Holmgren-Type Results Whern the Characteristic Set Contains an Involutive Manifold}

\section{$\S$ 4. Some Results of Direct Infinetisimal Geometry}

The following situation shall be frequently used

4.1.

$\Omega$ is an open set of $\mathbb{R}^{\nu}$, containing the point $x_{0} ; \phi(x)$ is a real, $C^{2}$ function defined in $\Omega$, satisfying $\phi\left(x_{0}\right)=0$ and $d \phi(x) \neq 0$ in $\Omega$.

$$
\xi_{0}=d \phi\left(x_{0}\right) \text { and } \Omega^{+}=\{x \in \Omega ; \phi(x)>0\}
$$

4.2. Definition: Cotangent normal bundle of an arbitrary closed set $F$ of $\mathbb{R}^{\nu}$. We say that $\left(x_{0}, \xi_{0}\right)$ belongs to $T_{F}{ }^{*} \mathbb{R}^{\nu}$ if $x_{0} \in F$ and if it is possible to find $\Omega$ and $\phi$ satisfying 4.1 such that $F \cap \Omega^{+}=\phi$.

We proved parts $\mathrm{a}$ and $\mathrm{b}$ of the following theorem in [2], [3], 
part $\mathrm{c}$ is due to Hörmander [10].

4.3. Theorem. Let $F$ be a closed set of $\boldsymbol{R}^{\nu}$.

a) Let $Z$ be a lipschitzian vector field, such that its principal symbol $z(x, \xi)$ vanishes on $T_{F}{ }^{*} \mathbb{R}^{\nu}$. Then, $F$ is a union of integral curves of $Z$.

b) Let $q_{1}(x, \xi)$ and $q_{2}(x, \xi)$ be homogeneous $C^{1}$ functions vanishing on $T_{F}{ }^{*} \mathbb{R}^{\nu}$. Then the Poisson bracket $\left\{q_{1}, q_{2}\right\}$ vanishes on $T_{F}{ }^{*} \mathbb{R}^{\nu}$.

c) Let $q(x, \xi)$ be a real homogeneous $C^{1}$ function vanishing on $T_{F}{ }^{*} \mathbb{R}^{\nu}$. Let $\left(x_{0}, \xi_{0}\right)$ be a point of $T_{F}{ }^{*} \mathbb{R}^{\nu}$ and let $(x(t), \xi(t))$ be the bicharacteristic with respect to $q$ starting from $\left(x_{0}, \xi_{0}\right)$. Then, $x(t)$ cannot go out of $F$ at the second order, i.e., if $\Omega$ and $\phi$ satisfy 4.1 with $F \cap \Omega^{+}=\phi$, it is impossible to have $\phi(x(t)) \geqq \alpha t^{2}$ with $\alpha>0$.

Classical Holmgren theorem asserts that, if $F$ is the support of a solution $u$ of $P\left(x, D_{x}\right) u=0$, then $T_{F}{ }^{*} R^{\nu}$ is contained in the characteristic set of $P$. In [2], [3], [10], it is shown that theorem 4.3 gives easily improvements of Holmgren theorem. Here, we shall apply theorem 4.3 to subsets of the cotangent budle.

\section{§5. Main Theorem}

We denote by $M=T^{*} R^{\nu}$ the cotangent bundle, and by $x^{*}=(x, \xi)$ points of $M$. Let $W$ be a regular involutive manifold (see $\S 1$ ) of codimension $n$. Then, there is a natural foliation of $W$ by $n$-dimensional leaves called $W$-bicharacteristic leaves.

The symplectic structure defines an isomorphism of $(\mathrm{TM})_{x_{0} *}$ and $\left(T^{*} M\right)_{x_{0} *}$ which induces the following isomorphism:

5.1.

$$
\left(T_{W} M\right)_{x_{0}^{*}}=\left(T^{*} \Sigma\right)_{x_{0}^{*}}
$$

where $x_{0}$ belongs to $W,\left(T_{W} M\right)_{x_{0} *}=(T M)_{x_{0} *} /(T W)_{x_{0}{ }^{*}}$ is the normal tangent space, and $\Sigma$ is the $W$-bicharacteristic leaf through $x_{0}$.

5.2. We assume now that the pseudo-differential operator $P$ satisfies $W \subset$ Char $(P)$. Let $k$ be the greatest integer such that $P_{\mu}$ and all its derivatives up to order $k-1$ vanish on $W$. 
5.3. Definition. (microcharacteristic tangent vector) Let $(\delta x, \delta \xi)$ $\in(T M)_{x_{0} *}$ with $x_{0}=\left(x_{0}, \xi_{0}\right) \in W$. We say that $(\delta x, \delta \xi)$ is microcharacteristic if

$$
P_{\mu}\left(x_{0}+\varepsilon \delta x, \xi_{0}+\varepsilon \delta \xi\right)=o\left(\varepsilon^{k}\right)
$$

It is easy to see that this property depends only on the class of $(\delta x, \delta \xi)$ in $\left(T_{W} M\right)_{x_{0} *}$

5.4. Definition. (microcharacteristic cotangent vector) Let $\Sigma$ be a W-bicharacteristic leaf, and $\left(x_{0}^{*}, \eta_{0}^{*}\right) \in T^{*} \Sigma$. We say that $\left(x_{0}^{*}\right.$, $\left.\eta_{0}{ }^{*}\right)$ belongs to Microchar $(P)$ if the tangent vector corresponding to $\eta_{0}{ }^{*}$ by isomorphism 5.1. is microcharacteristic as defined in 5.3.

We shall use the following notations to state the main theorem.

\section{5.}

$\Sigma$ is a W-bicharacteristic leaf, and $\left(x_{0}{ }^{*}, \eta_{0}{ }^{*}\right) \in T^{*} \Sigma$. $\omega$ is an open subset of $\Sigma$ containing $x_{0}$. The real, $C^{2}$ function $\varphi$ is defined on $\omega$, and satisfies $\varphi\left(x_{0}^{*}\right)=0$ and $d \varphi\left(x^{*}\right) \neq 0$ in $\omega$.

$$
\eta_{0}^{*}=d \varphi\left(x_{0}^{*}\right) \text { and } \omega^{+}=\left\{x^{*} \in \omega \mid \varphi\left(x^{*}\right)>0\right\} \text {. }
$$

5.6. Theorem. Assume 5.2 and 5.5, and let $u$ be a microfunction solution of $P u=0$. Assume moreover that $\left(x_{0}{ }^{*}, \eta_{0}{ }^{*}\right) \notin$ Microchar $(P)$. Then, $u=0$ in $\omega^{+}$implies $x_{0}^{*} \notin \operatorname{Supp}(u)$.

\section{§ 6. Applications to Propagation of Singularities and Uniqueness of Cauchy Problem}

Using results of $\S 4$, it is possible to give extensions of theorem 5.6 similar to extensions of Holmgren theorem. As a corollary, we shall obtain a new improvement of Holmgren theorem. We shall use following notations, where $\Sigma$ is a $W$-bicharacteristic leaf.

\section{1.}

$\mu \mathcal{I}$ : (microcharacteristic ideal) Set of real, homogeneous, $C^{\infty}$ functions $q\left(x^{*}, \eta^{*}\right)$ defined on $T^{*} \Sigma$ and vanishing on Microchar $(P)$. 
$\mathcal{L} \mu \mathcal{I}:$ Lie algebra (with respect to Poisson bracket) generated by $\mu \mathcal{G}$.

$\mathcal{L}^{*}$ Microchar $(P)$ : Set of $\left(x^{*}, \eta^{*}\right) \in T^{*} \Sigma$ such that $r\left(x^{*}, \eta^{*}\right)=0$ for each $r$ belonging to $\mathcal{L} \mu \mathcal{G}$.

6.2. Theorem. Let $u$ be a microfunction solution of $P u=0$.

a) Let $Z$ be a vector field on $\Sigma$ such that its principal symbol $z\left(x^{*}\right.$, $\left.\eta^{*}\right)$ belongs to $\mathcal{L} \mu \mathcal{G}$. Then, $\operatorname{Supp}(u)$ is a union of integral curves of $Z$.

b) With notations 5.5, assume that $\left(x_{0}{ }^{*}, \eta_{0}{ }^{*}\right) \notin \mathcal{L}^{*} \operatorname{Microchar}(P)$. Then, $u=0$ in $\omega^{+}$implies $x_{0}{ }^{*} \notin \operatorname{Supp}(u)$.

c) Assume $\left(x_{0}{ }^{*}, \eta_{0}{ }^{*}\right) \notin \mathcal{L}^{*} \operatorname{Microchar}(P)$, and that there exist $r \in \mathcal{L} \mu \mathcal{I}$ such that the bicharacteristic with respect to $r$ starting from $\left(x_{0}{ }^{*}, \eta^{*}\right)$ goes into $\omega^{+}$at the second order. Then, $u=0$ in $\omega^{+}$implies $x_{0}{ }^{*} \notin \mathrm{Supp}$ (u).

The simplest application holds under the following assumptions.

6.3. Char $(P)$ is an analytic manifold of codimension $d$, defined by $q_{1}$ $(x, \xi)=\cdots=q_{d}(x, \xi)=0$, with $d q_{1}, \cdots, d q_{d}$ independant on $\operatorname{Char}(\mathrm{P})$.

6.4. $\mathcal{L}^{*} \operatorname{Char}(P)=W$ (the set of common zeroes of the Lie algebra generated by $\left.q_{1}, \cdots, q_{d}\right)$ is an analytic manifold of codimension $n$, defined by $r_{1}(x, \xi)=\cdots=r_{n}(x, \xi)=0$, with $d r_{1}, \cdots, d r_{n}, \Sigma \xi_{i} d x_{i}$ independent on $W$. 6.5. The principal symbol $P_{\mu}$ vanishes on $\operatorname{Char}(\mathrm{P})$ exactly at order $k$, i.e. $\left|P_{\mu}(x, \xi)\right| \geqq C \Sigma\left|q_{i}(x, \xi)\right|^{k}$ with $C>0$, near $\operatorname{Char}(\mathrm{P})$.

6.6. Theorem. Assume 6.3, 6.4, 6.5, and let $u$ be a microfunction solution of $P u=0$. Then, the support of $u$ is a union of $W$ bicharacteristic leaves.

This is an easy consequence of theorem 6.2, after noting that the principal symbols of hamiltonian fields $H_{q_{i}}$ and $H_{r_{j}}$ belong respectively to $\mu \mathcal{I}$ and $\mathcal{L} \mu \mathcal{G}$.

6.7. Corollary. Assume that $P$ is a differential operator satis- 
fying 6.3, 6.4, 6.5, and let $u$ be a hyperfunction solution of $P u=0$.

With notations 4.1. suppose that $\left(x_{0}, \xi_{0}\right) \in \mathcal{L}^{*}$ Char $(P)$ and that there exist $r(x, \xi)$, vanishing on $\mathcal{L}^{*}$ Char $(P)$ such that the bicharacteristic with respect to $r$, starting from $\left(x_{0}, \xi_{0}\right)$ goes into $\Omega^{+}$before going out of $\Omega$. Then, $u=0$ in $\Omega^{+}$implies $u=0$ near $x_{0}$.

Under the regularity assumptions 6.3 to 6.5 , this result improves the result of Hörmander in [10], which is valid without these assumptions.

\section{§ 7. Sketch of the Proof of Theorem 5.6}

Using a quantized contact transform, and changing notations ( $\mathbb{R}^{\nu}$ $=\mathbb{R}_{x}{ }^{n} \times \mathbb{R}_{t}{ }^{p}$, cf. $\S 1$ ) we are reduced to the following case:

$$
\begin{aligned}
& P\left(x, t, D_{x}, D_{t}\right)=\sum_{|\lambda|=k} A_{\lambda}\left(x, t, D_{x}, D_{t}\right) D_{x}{ }^{\lambda}+\text { lower order terms, } \\
& W=\{(x, t, \xi, \tau) \mid \xi=0\} ; x_{0}^{*}=\left(0,0,0, \tau_{0}\right) ; \tau_{0}=(1,0, \cdots, 0) \\
& \sum a_{\lambda}(x, t, 0, \tau) \xi^{\lambda} \neq 0 \text { for } \xi \neq 0 \text { and } \xi_{i} \geqq 0, i=1, \cdots, n,
\end{aligned}
$$

where $a_{\lambda}$ is the principal symbol of zero-order pseudo-differential operator $A_{\text {r. }}$

We consider the $2^{n}$ following cones in $\mathbb{R}^{n}$ :

$$
\alpha \in\{-1,1\}^{n} ; \Gamma_{\alpha}=\left\{x \in \mathbb{R}^{n} \mid \alpha_{i} x_{i}>0, i=1, \cdots, n\right\}
$$

Putting $\alpha_{+}=(1, \cdots, 1)$ and $\alpha_{-}=(-1, \cdots,-1)$, using the decomposition, existence, and extension arguments of [6], we obtain:

7.1. Theorem. Let $u$ be a microfunction defined near $x_{0}{ }^{*}$, satisfying $P u=0$. It is then possible to find holomorphic functions $f_{\alpha}$, defined in the intersection of $\mathbb{R}^{n+p}+i\left(G+\Gamma_{\alpha}\right)$ with a neighbourhood of 0 , where $G$ is a convex cone of $\mathbb{R}^{n+p}$ the polar set of which is a small neighbourhood of $\left(0, \tau_{0}\right)$ such that

$$
u=\sum_{\alpha \neq \alpha_{+}, \alpha_{-}} b\left(f_{\alpha}\right) \text { near } x_{0}^{*}
$$

Recall now briefly Kashiwara's theory of microlocalization of sheaf $\mathscr{C}_{n}$. We denote by $E$ the space $\mathbb{R}^{n+p} \times \mathbb{S}^{n+p-1}$, and by $N$ the subspace of $E$ defined by $\xi=0$. We have $N=\mathbb{R}^{n+p} \times S^{p-1}$. 
The sheaf $\mathscr{C}$ is defined on $E$, and the sheaf $\mathscr{C}_{h}$ of microfunctions $u$ on $\mathbb{C}^{n} \times \boldsymbol{R}^{p}$ satisfying $\partial / \partial \bar{z}_{j} u=0$ is a sheaf on $\widetilde{E}=\boldsymbol{C}^{n} \times \boldsymbol{R}^{p} \times S^{2 n+p-1}$ with support in $\mathbb{C}^{n} \times \boldsymbol{R}^{p} \times \boldsymbol{S}^{p-1}$. We denote by $\pi$ the projection $N \times \boldsymbol{S}^{n-1} \rightarrow N$.

7.2. (Kashiwara) [11][12]. There exists a sheaf $\mathscr{C}_{N}{ }^{-}$on $N \times S^{n-1}$ such that the following diagram is exact

$$
\left.0 \longrightarrow \mathscr{C}_{h}\right|_{N} \longrightarrow \mathcal{H}_{N}{ }^{n}\left(\widetilde{E}^{\downarrow} \mathscr{\mathscr { C }}_{h}\right) \stackrel{S_{p}}{\longrightarrow} \pi_{*}\left(\mathscr{C}_{N}{ }^{-}\right) \longrightarrow 0
$$

and that, if $f$ is holomorphic near 0 in $\boldsymbol{R}^{n+p}+i(G+\Gamma)$, with $G$ as in theorem 7.1 and $\Gamma$ an open convex cone in $\boldsymbol{R}^{n}$, we have

7.3. Supp $(\operatorname{Sp} \circ \mathrm{j} \circ \mathrm{b}(f)) \subset N \times \Gamma^{\circ}$

7.4. Theorem (Kashiwara) Let $u$ be a microfunction on $\boldsymbol{R}^{n+p}$, defined near $\left(0,0,0, \tau_{0}\right)$ such that points $\left(\left(0,0, \tau_{0}\right) ;(0, \cdots, \pm 1)\right)$ do not belong to the support of $S p \circ j(u)$. Then, if $u$ vanishes for $x_{n}<0$, we have $u=0$, near $\left(0,0,0, \tau_{0}\right)$.

Theorem 5.6 is an easy consequence of 7.1, 7.3 and 7.4. In some sense, 7.4 is a double microlocalization of unique continuation principle for analytic functions, while 7.1 is a double microlocalization of analyticity of solutions of elliptic equations.

\section{References}

[1] Bros, J. and Iagolnitzer, D., Support essentiel et structure analytique des distributions, Séminaire Goulaouic-Lions-Schwartz no 18 (1974-1975).

[2] Bony, J.-M., Principe du maximum, inégalité de Harnack et unicité du problème de Cauchy pour les opérateurs elliptiques dégénérés, Ann. Inst. Fourier Grenoble 19-1 (1969), 277-304.

[3] Bony, J.-M., Une extension du théoreme de Holmgren sur l'unicité du problème de Cauchy, C. R. Acad. Sc. Paris 268 (1969), 1103-1106.

[4] Bony, J.-M., Propagation des singularités différentiables pour des opérateurs à coefficients analytiques, Astérisque 34-35 (1976), 43-91.

[5] Bony, J.-M., Extensions du théorème de Holmgren, Séminaire Goulaouic-Schwartz, 
no 17 (1975-1976).

[6] Bony, J.-M. and Schapira, P., Propagation des singularités analytiques pour les solutions des équations aux dérivées partielles, Ann. Inst. Fourier Grenoble 26-1 (1976), 81-140.

[7] Duistermaat, J., and Hörmander, L., Fourier integral operators II, Acta Math. 128 (1972), 183-269.

[8] Hörmander, L., Fourier integral operators I, Acta Math. 127 (1971), 79-183.

[9] Hörmander, L., Uniqueness theorems and wave front sets for solutions of linear partial differential equations with analytic coefficients, Comm. Pure Appl. Math. 23 (1970), 329-358.

[10] Hörmander, L., A remark on Holmgren's uniqueness theorem, J. Diff. Geometry 6 (1971), 129-134.

[11] Kashiwara, M., Lecture in colloquium Hyperfonctions et Physique theorique, Nice (1973) (unpublished).

[12] Kashiwara, M., Oral communications, Nice (1973), Paris (1975).

[13] Sato, M., Kawai, T. and Kashiwara, M., Hyperfunctions and pseudo-differential equations, Lecture Notes in Math. 287 (1973), 265-529.

[14] Sjöstrand, J., Propagation of singularities for operators with multiple involutive characteristics, Ann. Inst. Fourier Grenoble 26-1 (1976), 141-155. 
